www.jmscr.igmpublication.org

Impact Factor (SJIF): 6.379

Index Copernicus Value: 71.58

ISSN (e)-2347-176x ISSN (p) 2455-0450

crossref DOI: _https://dx.doi.org/10.18535/jmscr/v6i3.08

Journal Of Medical Science And Clinical Research

\title{
Study of prognostic factors and clinical outcome of Acute Pancreatitis patients with routine investigations in a Teaching Hospital
}

\author{
Authors \\ Dr M Basavarajappa ${ }^{1}$, Dr Ananth S Mathad ${ }^{2}$ \\ ${ }^{1}$ Professor, Dept of General Surgery, SSIMS\&RC, Davangere \\ ${ }^{2}$ Postgraduate, Dept of General Surgery, SSIMS\&RC, Davangere
}

\begin{abstract}
This tertiary care hospital based retrospective study was performed to find out the prognostic factors and clinical outcome of acute pancreatitis from the available clinical, biochemical, haematological and radiological data. A total of 307 cases were studied. Peak incidence was in the fourth decade. Among the known aetiological factors $18 \%$ were due to gallstone disease, $10 \%$ alcoholism, $4 \%$ viral, but $60 \%$ were idiopathic. In this study $82 \%$ patients were had mild and $18 \%$ had severe acute pancreatitis. Overall mortality rate was $6 \%$, mortality was significantly higher (33.33\%) in severe acute pancreatitis $(33.3 \%$ vs 0\%). A higher mortality was associated with concomitant medical or surgical diseases, leucocytosis. Other factors associated with a higher mortality were high blood glucose, serum creatinine level and a lower serum albumin and calcium level. This study highlights importance of routine investigations and guides the primary care hospital for early intervention and referral for higher centre.

Keyword: Acute Pancreatitis, Prognosis, Mortality.
\end{abstract}

\section{Introduction}

Acute pancreatitis is an acute inflammatory process of the pancreas with variable involvement of regional tissues and remote organ systems. ${ }^{1}$ The incidence of pancreatitis varies in different countries and depends on cause, e.g., alcohol, gallstones, metabolic factors and viral infections. The estimated incidence in industrialized countries is 10-20/ 100,000 per year ${ }^{2}$. The mortality is less than $1 \%$ in mild Acute Pancreatitis with mortality upto $10-20 \%$ in severe acute pancreatitis. The average mortality rate in severe acute pancreatitis approaches $2-10 \%{ }^{3}$

There are many causes of acute pancreatitis, but the mechanisms by which these conditions trigger pancreatic inflammation have not been identified.
Gall stones and chronic ethanol abuse account for $70 \%$ of cases of acute pancreatitis. ${ }^{4}$

Approximately 2 to $5 \%$ of cases of acute pancreatitis are drug-related. Drugs cause pancreatitis either by a hypersensitivity reaction or by the generation of a toxic metabolites. Viral infections such as mumps and coxsackie $B$ virus plays the etiological factors in a acute pancreatitis. Other causes include trauma, after biliary, gastric, splenic surgery, ERCP, hyperparathyroidism, hypercalcaemia, hyperlipidaemia, Diabetes, porphyria, autoimmune diseases, vascular diseases, idiopathic, scorpion venom, Biliary ascariasis, Clonorchissinensis, Mycoplasma pneumonia, Infectious mononucleosis are found to be the etiological factors in lesser population. ${ }^{5}$ 
Early accurate diagnosis is very important for its management. Symptoms of acute pancreatitis vary considerably. Some patients have mild epigastric discomfort that may mimic peptic ulcer. Others suffer from an acute catastrophic illness that cannot be readily distinguished from such severe intra- abdominal condition as perforated duodenal ulcer or mesenteric infarction. For this reason the clinician must carefully evaluate information derived from other sources that supplement the history and physical examination including laboratory tests, imaging studies before arriving at a correct diagnosis of acute pancreatitis.

Removal of factors that may have precipitated the attack is one of the important aspects of management of acute pancreatitis. The first attacks are usually severe, although subsequent ones tend to be mild. If the cause of the attack can be eliminated there will be no further attacks and the pancreas will return to normal in terms of its morphology and function. If the cause of the attack is not eliminated then leads to recurring episodes of inflammation cause progressive destruction of the gland, leading to chronic pancreatitis or chronic relapsing pancreatitis. ${ }^{6}$

The variation in the etiology of pancreatitis from series to series is quite marked and depend on the country of origin ${ }^{7}$. In this potentially fatal disease, mortality depends on the severity. Most attacks of acute pancreatitis are mild and death is unusual in such patients. Mortality and morbidity is very high in those with severe acute pancreatitis. So grading of severity is of critical importance in providing appropriate therapy in acute pancreatitis. Studies suggest that outcome of an attack of acute pancreatitis can be improved by early treatment and therefore, urgent treatment of severe acute pancreatitis will reduce morbidity and mortality ${ }^{8}$.

Many attempts have been made to improve the recognition of a severe attack at the onset. Combinations of clinical and laboratory data that can indicate the severity of acute pancreatitis within first 48 hours after admission have been developed by Ranson and colleagues and clinician from Glasgow. APACHE II illness grading system can also be used to predict severity ${ }^{9}$. All those systems are cumbersome and such combination system has low sensitivity in clinical practice ${ }^{10}$.

Presence of SIRS (Systemic inflammatory response syndrome), scores such as the Ranson, the Glasgow, and Acute Physiology and Chronic Health Evaluation (APACHE) are practical for assessing the severity of the disease but are not sufficiently well validated for predicting mortality. Early organ dysfunction predicts disease severity and patients require early intensive care treatment. Antibiotic prophylaxis is usually ineffective and early enteral feeding results in reduction of local and systemic infection. Management of acute pancreatitis has changed significantly over the past years. Early management is nonsurgical, solely supportive and patients with infected necrosis with worsening sepsis need intervention. Early intensive care has definitely improved the outcome of patients. ${ }^{11}$ Genetic polymorphisms and mutations also contribute to difficulty in predicting the outcome. ${ }^{12}$

A number of risk factors have been evaluated in the clinical setting. These include markers of pancreatic injury and of inflammatory response, together with simple routine tests and imaging methods ${ }^{13}$. However, these markers are not available in every hospital and some of them proved not to be useful in current clinical practice. The main objective of this study was to observe the prognostic factors and clinical outcome of acute pancreatitis with routine investigations.

\section{Material and Methods}

This was a retrospective study carried out at Department of Surgery, S.S institute of Medical Sciences and research centre from January 2014 to December2016. In this study a total 307 cases of acute pancreatitis were analysed.

Patients were selected using the inclusion criteria of appropriate clinical evidence, associated with an elevation of serum amylase and serum lipase of age more than 18 years and irrespective of sex admitted under surgery department. Normal value 
of serum amylase was accepted upto220 U/L and that of urinary amylase was accepted upto 400U/L.

Exclusion criteria were a serum amylase level greater than upper limit of normal value with a diagnosis of pancreatic or periampulary cancer, chronic pancreatitis, perforation of the gut, and diabetic ketoacidosis.

A predesigned case record form was used and detailed clinical history was recorded from the patient's history or written history sheet. Age, sex, address, symptoms and signs were noted.

Relevant past history, family history and personal history, especially alcohol consumption were recorded. Associated medical diseases like diabetes mellitus, hypertension, chronic renal failure, bronchial asthma, chronic obstructive pulmonary disease (COPD) and ischemic heart disease were also noted.

The most likely aetiological factor was identified by analysing history, physical examination and relevant investigations. Gall stone related acute pancreatitis was based upon the identification of stone in the gall bladder or in the common bile duct (CBD) by sonography. Alcohol, viral or trauma was considered to be aetiological factor from the history. When no known cause was found it was termed idiopathic.

Criteria of severity were based on the Glasgow criteria. To evaluate prognostic factors, a standard scoring system like Ranson, Glasgow or APACHE-II, all data were not available. Available data of all the studied patients were analysed to find out the important prognostic factors. Data of survivors and non-survivors were compared-two demographic (age and sex), two haematological (haemoglobin and total white cell count), five biochemical (blood sugar, serum bilirubin, aspartate aminotransferase, serum albumin and serumcalcium) parameters. Blood glucose level in those patients previously diagnosed diabetic were not analysed for the prognostic purpose. Patients were stratified in four age groups (i.e., below 20 years, 20 to 40 years, 41 to 60 years and above 60 years).

Survivors were defined as patients discharged alive from the hospital and non-survivors as those who died from acute pancreatitis or its complications during hospitalization.

\section{Result}

Of the 307 patients studied, 193 patients were male and 114 were female.

A age was 18 to 74 years, with a mean age of 35 years. Out of 307 patients, 67 (22\%) had gallstone related pancreatitis. Alcoholism was found in $31(10 \%)$ cases, trauma in $6(2 \%)$ case, viral etiologyin $12(4 \%)$, hypertriglyceridemia in 6 (2\%) cases. No cause was foundin $183(60 \%)$ cases.

Table-1 Ultrasonographic findings of acute pancreatitis ( $\mathrm{N}=307)$

\begin{tabular}{|l|cc|cc|}
\hline & \multicolumn{2}{|c|}{ MALE } & \multicolumn{2}{c|}{ FEMALE } \\
\hline USG FINDINGS & TOTAL & PERCENTAGE & TOTAL & PERCENTAGE \\
\hline SWOLLEN/ENLARGED PANCREAS & 92 & $47.67 \%$ & 20 & $17.54 \%$ \\
\hline NORMAL PANCREAS & 42 & $21.76 \%$ & 39 & $34.21 \%$ \\
\hline CHOLELITHIASIS & 35 & $18.13 \%$ & 39 & $34.21 \%$ \\
\hline CHOLEDOCHOLITHIASIS & 8 & $4.15 \%$ & 8 & $7.01 \%$ \\
\hline ASCITES & 16 & $8.29 \%$ & 8 & $7.01 \%$ \\
\hline
\end{tabular}

In Table-I, above table shows the statistical representation of ultrasonographic evaluation of acute pancreatitis patients. It revealed 112 $(36.48 \%)$ patients had swollen or enlarged pancreas. Cholelithiasis were identified in 74 (35.12) patients, choledocholithiasis in $16(5.2 \%)$ patients. Pancreas sonologically appeared normal in $81(42.12 \%)$ patients. 
Table-II Hematological profile of acute pancreatitis $(\mathrm{N}=307)$

\begin{tabular}{|l|cc|rc|}
\hline & \multicolumn{2}{|c|}{ MALE } & \multicolumn{2}{c|}{ FEMALE } \\
\hline PARAMETERS & TOTAL & PERCENTAGE & TOTAL & PERCENTAGE \\
\hline $\mathrm{Hb} \% \geq 10 \mathrm{gm} / \mathrm{dl}$ & 169 & $88.0 \%$ & 95 & $84 \%$ \\
\hline $\mathrm{Hb} \% \leq 10 \mathrm{gm} / \mathrm{dl}$ & 24 & $12.0 \%$ & 19 & $16 \%$ \\
\hline TLC OF $\mathrm{WBC} \geq 11000 / \mathrm{mm}^{3}$ & 73 & $38.0 \%$ & 18 & $16 \%$ \\
\hline TLC $\mathrm{OF} \mathrm{WBC} \leq 11000 / \mathrm{mm}^{3}$ & 120 & $62.0 \%$ & 96 & $84 \%$ \\
\hline
\end{tabular}

(Table-II). Of the two heamatological parameters tested, haemoglobin level was $10 \mathrm{gm} / \mathrm{dl}$ or above were $264(86 \%)$ patients and $7(14 \%)$ patients were below this level. TC of $\mathrm{WBC}>15000 / \mathrm{mm}^{3}$

was in $15(30 \%)$ patients but 35 patients were below this level. Out of two haematological parameters tested a higher leucocyte count and haemoglobin was associated with high mortality.

Table-III Bio-Chemical profile of acute pancreatitis $(\mathrm{N}=307)$.

\begin{tabular}{|c|c|c|c|c|}
\hline & \multicolumn{2}{|c|}{ MALE } & \multicolumn{2}{|c|}{ FEMALE } \\
\hline PARAMETERS & TOTAL & PERCENTAGE & TOTAL & PERCENTAGE \\
\hline RBS $\geq 110 \mathrm{mg} / \mathrm{dl}$ & 20 & $10 \%$ & 12 & $10 \%$ \\
\hline RBS $\leq 110 \mathrm{mg} / \mathrm{dl}$ & 173 & $90 \%$ & 102 & $90 \%$ \\
\hline S.Bilirubin( $(\geq 1.2 \mathrm{mg} / \mathrm{dl})$ & 10 & $5 \%$ & 6 & $5 \%$ \\
\hline S.Bilirubin( $\leq 1.2 \mathrm{mg} / \mathrm{dl})$ & 183 & $95 \%$ & 108 & $95 \%$ \\
\hline S.ALT $(\geq 45 I U / L)$ & 20 & $10 \%$ & 6 & $5 \%$ \\
\hline S.ALT( $\leq 45 I U / L)$ & 173 & $90 \%$ & 108 & $95 \%$ \\
\hline S.Albumin $(\geq 3.2 \mathrm{~g} / \mathrm{dl})$ & 181 & $94 \%$ & 101 & $89 \%$ \\
\hline S.Albumin $(\leq 3.2 \mathrm{~g} / \mathrm{dl})$ & 12 & $6 \%$ & 13 & $11 \%$ \\
\hline S.Calcium( $(\geq 8.5 \mathrm{mg} / \mathrm{dl})$ & 181 & $94 \%$ & 101 & $89 \%$ \\
\hline S.Calcium $(\leq 8.5 \mathrm{mg} / \mathrm{dl})$ & 12 & $6 \%$ & 13 & $11 \%$ \\
\hline
\end{tabular}

Table III showing low serum calcium and albumin aspartate aminotransferase were found not to and a high blood glucose level were associated associated with increased mortality.

with higher mortality. Serum bilirubin and

Table-IV Complication Profile of acute pancreatitis $(\mathrm{N}=307)$

\begin{tabular}{|l|ccc|cc|}
\hline & \multicolumn{3}{|c|}{ Male } & \multicolumn{2}{c|}{ Female } \\
\hline Complication & Total & Percent & Total & Percent \\
\hline Hyperglycemia & $\mathbf{1 2}$ & $\mathbf{6}$ & $\mathbf{7}$ & $\mathbf{6}$ \\
\hline Hypocalcemia & $\mathbf{6}$ & $\mathbf{3}$ & $\mathbf{1 9}$ & $\mathbf{1}$ \\
\hline Pseudocyst & $\mathbf{1 7}$ & $\mathbf{9}$ & $\mathbf{2}$ & $\mathbf{2 3}$ \\
\hline Ascites with pleural effusion & $\mathbf{1 2}$ & $\mathbf{6}$ & $\mathbf{2 6}$ & $\mathbf{5}$ \\
\hline Upper gi bleeding & $\mathbf{6}$ & $\mathbf{3}$ & $\mathbf{6}$ & $\mathbf{3}$ \\
\hline Obstructive Jaundice & $\mathbf{2}$ & $\mathbf{1}$ & $\mathbf{4}$ & \\
\hline
\end{tabular}

In this study 119 (38\%) patients developed complications. Out of these 20 patients, 55 patients were male and 64 patients were female. comparison to males i.e, hypocalcemia, ascites, pleural effusion and obstructive jaundice. (TableIV).

Complication rate were more in females than in

Table-V Prognostic profile of acute pancreatitis (Male=193), (Female-114)

Male

\begin{tabular}{|l|c|c|c|c|c|c|}
\hline Prognosis & $<20$ & $21-40$ & $41-60$ & $>60$ & Total & Percentage \\
\hline Survivour & 10 & 120 & 38 & 13 & 181 & 93.75 \\
\hline Non-Survivour & - & 6 & 6 & - & 12 & 6.25 \\
\hline
\end{tabular}

Female

\begin{tabular}{|l|c|c|c|c|c|c|}
\hline Prognosis & $<20$ & $21-40$ & $41-60$ & $>60$ & Total & Percentage \\
\hline Survivour & 6 & 54 & 30 & 17 & 107 & 94.44 \\
\hline Non survivor & - & 3 & - & 4 & 7 & 5.56 \\
\hline
\end{tabular}


Out of 307 patients, 41 (82\%) were graded as mild and $9(18 \%)$ as severe. A total $19(6 \%)$ patient died. A significantly higher mortality was found in patients with severe pancreatitis, than those with mild attack (0\%). Mortality is more at the early age group in males whereas in females it was found to be in latter age group.

\section{Discussion}

It was retrospective study to evaluate 307 cases of acute pancreatitis with the basic clinical, biochemical, haematological parameter for prognostic outcome and clinical outcome in tertiary care hospital. Only those cases who were inpatient at SSIMS \&RC were subjected to study.

Acute pancreatitis is a disease that varies in severity ranging from a mild and self limited illness to a very severe and rapidly progressive condition leading to multiple organ failure and eventually to death. ${ }^{14}$ An early diagnosis and identification of those who are at risk of development of severe disease and rapid institution of therapy might reduce in morbidity and mortality. ${ }^{15}$

If the aetiological factor/factors are known and can be eliminated, further attacks can also be prevented. In this study the age range of patient was 13 to 74 years with a peak incidence is in the fourth decade. In western countries the peak incidence of alcoholic pancreatitis is in the fifth decade, whereas pancreatitis associated with gallstones and other cases peaks in the seventh decade. $^{16}$

As we are in developing country the lower age incidence of acute pancreatitis may be due to lower socioeconomic status and low literacy level at rural area, occurrence of gallstone disease in the female in early age due to early and multiple pregnancy.

In female it is more commonly related to gallstone disease. In this study there is male predominance (Male: Female = 1.78:1).

In different series of the USA, Western Europe and Asia, gallstones are the most common cause of acute pancreatitis accounting for approximately
$45 \%$ cases. Alcohol is the second most common cause. However, the variation of aetiology of pancreatitis is quite marked and depend on country of origin. ${ }^{17}$

In this study most cases (60\%) were idiopathic i.e. no cause is found. These cases are probably related to viral or bacterial infection which needs further exploration. Gallstone ranks the second among the known aetiological factors (35\%cases). This high incidence of idiopathic acute pancreatitis in our country is due to higher prevalence of infections because of poor nutrition, poor sanitation, overcrowding and lack of knowledge. In our series only $10 \%$ cases were due to alcohol. This low incidence in contrast to Western countries reflects the socio-cultural and religious factors that discourage alcohol consumption in our country. Mumps is a rare cause of acute pancreatitis in developed countries. $^{18}$

But in our study it seems to be an important aethological factors accounting for $4 \%$ cases. This higher incidence may due to overcrowding. Blunt trauma to the abdomen is a recognized cause of acute pancreatitis, which results from the disruption of the pancreatic ductal system. Blunt trauma from road traffic accidents is the dominant cause in Europe while direct injury by a knife or bullet is more common in North America. ${ }^{19}$

In this series there were only $1(2 \%)$ case which was caused by blunt trauma to abdomen. Hypertriglyceridemia was responsible in 1(2\%) case. Triglyceridemia levels exceeding > 880 $\mathrm{mg} / \mathrm{dl}$ increases the risk of pancreatitis. In this study $1(2 \%)$ case developed pancreatitis due to hypertriclyceridemia which is consistent with that of other countries. ${ }^{20}$

In this study a large group of patient, no cause could be identified. This so called idiopathic cause is the most common cause of acute pancreatitis in most Western series.

Recently, two prospective studies on consecutive patients with apparently idiopathic pancreatitis showed that two-thirds to three-fourths had microlithiasis as the presumed cause, as 
documented by biliary drainage studies, follow-up sonogram and ERCP with sphinterotomy or cholecytectomy. ${ }^{21}$

No such data was available in the hospital documents of studied patients in this series. All patients of this series underwent ultrasonographic examinations. More than $46 \%$ of patients had sonographic abnormalities of pancreas. Fourty-six (46\%) percent pancreas appeared to be normal which may be due to poor visualization of the gland. Despite remarkable advances in the understanding of pathophysiology of acute pancreatitis and advances in supportive and intensive care, the overall mortality rate for patients with acute pancreatitis has remained in the range of $10 \%$ for over last $40 \mathrm{yrs}^{22}$

A large prospective study showed that 3 of every 4 patients with acute pancreatitis will have a mild form of the disease which promptly responds to supportive measure. The fourth patient, however, will have a severe episode and approximately half of those patients will suffer from a complication and one third of them will die. ${ }^{23}$

In our series, 41(82\%) patients had mild and 9 (18\%) had severe acute pancreatitis with an overall mortality rate of $6 \%$. Now-a-days infectious complications of acute pancreatitis account for more than $80 \%$ of all death from this disease. $^{24}$

It seems clear, that if the mortality rate in acute pancreatitis is to be reduced, attention must be directed toward the identification and management of patients with various complications of infections. Early identification of these patients at increased risk for the development of complications may have significant therapeutic implications.

Careful and repeated clinical evaluation is clearly of paramount importance in the management of individual patients. These include age, fever, physical abnormality of the chest, a palpable mass and detectable intra abdominal fluid. Increased age is associated with a bad prognosis has been widely recognized and simply reflect a decreased physical reserve in the older population. In
Ranson's experience the mean age of patients who died and of those who had a complicated clinical course were significantly higher than those who recovered eventfully. ${ }^{25}$

Fan et al. have observed a mortality rate of $21.3 \%$ in patients over $75 \mathrm{yrs}$ old and $5.9 \%$ in the younger population. ${ }^{26}$

The high mortality was accounted for by a higher incidence of death related to concomitant medical or surgical diseases in the same hospital admission rather than to complications resulting directly from the pathological process of acute pancreatitis. When only deaths due to complications of acute pancreatitis were analyzed, the mortality rate was not significantly different between the young and elderly groups.

The reported relationship between patients sex and prognosis for acute pancreatitis varies. No relationship between the sex of the patients and prognosis has been demonstrated by Ranson and Brisando and our result also supported it. ${ }^{27}$

The overall white cell count may reflect the severity of the inflammation and a relationship between the degree of early leucocytosis and the severity of the pancreatitis has been noted by many authors. ${ }^{28}$

In this study, a higher total white cell count and low haemoglobin was associated with higher mortality and among the biochemical parameters tested a high blood sugar and serum creatinine level and a lower serum albumin and calcium levels were significantly associated with a higher mortality.

However, this study did not show any relationship between serum bilirubin, aspartate transaminase (AST), alkaline phosphatase, serum potassium levels and a higher mortality.

A higher aspartate transaminase (AST) and hypokalaemia were not found to be associated with a higher mortality rate. But in Ranson's and Brisando's experience a high blood sugar, serum creatinine and asparatate transaminase and low serum calcium and albumin were associated with poor prognosis. 
Concomitant medical diseases like diabetes mellitus, bronchial asthma, COPD, CRF and fever $>100^{\circ} \mathrm{F}$ within two days of onset of abdominal pain should be considered as poor prognostic factors, similarly a white blood cell count $>15,000 / \mathrm{mm} 3$, a high serum creatinine and a low serum albumin, calcium and presence of pleural effusion should also be considered as poor prognostic factors and such patients should be in managed in a high dependency or intensive care unit.

\section{Conclusion}

Acute pancreatitis is a neglected disease in our country. We are not aware of the actual scenario regarding the incidence, prevalence, cause or mortality rate of this treatable disease. Early recognition of bad prognostic factors can result in appropriate and timely management of acute pancreatitis.

\section{References}

1. Bradley El. $3^{\text {rd }}$ A clinically based classification system for acute pancreatitis, Atlanta, Ga, September 11 through 13, 1992. Arch surg. 1993;128:586.

2. Sargen K, Kingsnorth A. Management of Gallstone Pancreatitis: Effects of Deviation from Clinical Guidelines. J Pancreas (Online) 2001; (5):317 -322.

3. Singh VK, Bollen TL, Wu BU, et al. An assessment of the severity of interstitial pancreatitis. ClinGastroenterolHepatol 2011; 9:1098.

4. Corfield AP, Cooper Ml, Williamson RCN. Acute pancreatitis: A lethal disease of increasing significance. Gut. 1985;26:724.

5. Zheng $\mathrm{Y}$, Zhou $\mathrm{Z}$, Li $\mathrm{H}$, et al. A multicenter study on etiology of acute pancreatitis in Beijing during 5 years.Pancreas.2015 Apr;44(3):409-14.

6. Beger HG, Rau BM. Severe acute pancreatitis: Clinical course and management. World $\mathrm{J}$ Gastroenterol 2007;13:5043.

7. Wyncell DL. The management of severe acute necrotizing pancreatitis: an evidencebased review of the literature.Intensive Care Med 1999; 25: 146.

8. Steinberg WT. Acute pancreatitis. N Engl J Med 1994; 330:1198

9. Forsmark CE, Baillie J, AGA Institute Clinical Practice and Economics Committee, AGA Institute Governing Board. AGA Institute technical review on acute pancreatitis. Gastroenterology 2007;132:2022.

10. Tenner S, Baillie J, DeWitt $\mathrm{J}$, et al. American College of Gastroenterology guideline: management of acute pancreatitis. Am J Gastroenterol 2013; 108:1400.

11. Chauhan S, Forsmark CE. The difficulty in predicting outcome in acute pancreatitis. Am J Gastroenterol 2010; 105:443.

12. Johnson CD, Abu-Hilal M. Persistent organ failure during the first week as a marker of fatal outcome in acute pancreatitis. Gut 2004; 53:1340.

13. Dervenis C, Bassi C. Evidence-based assessment of severity and management of acute pancreatitis. Br J Surg 2000; 87:257258.

14. Beger HG, Rau B, Mayer J, Pralle U. Natural course of acute pancreatitis. WorldJ Surg. 1997;21:130-5.

15. Dervenis C, Johnson CD, Bassi C. Diagnosis, objective assessment of severity, and management of acute pancreatitis. Int J Pancreatol. 1999;25:195210.

16. Mayor AD, McMahon MJ. Gallstones and acute pancreatitis- is the association underestimated? Br J Surg. 1984;71:905.

17. Corfield AP, Cooper Ml, Williamson RCN. Acute pancreatitis: A lethal disease of increasing significance. Gut. 1985;26:724. 
18. M. Economou, M. Zissis. Infectious cases of acute pancreatitis. Annals of Gastroenterology 2000;13(2):98-101

19. Ranson JHC. Prognostic signs and the role of operative management in acute pancreatitis. SurgGynoclObstet 1994;139: 69-81

20. Funnell IC, Bomman PC, Weakley SP. Obesity: An important prognostic factor in acute pancreatitis. Br I Surg. 1993;80:484.

21. Houssin D, Castain GD, Lemoine J, Bismuth H. Microlithiasis of gall bladder. SurgGynecol Obstet. 1983;157:20-4.

22. Balthazar EJ, Freeny PC, van Sonnenberg E. Imaging and intervention in acute pancreatitis. Radiology. 1994;193:297306.

23. Kanus WA, Draper EA, Wagner DP, Zimmerman JE. APPACHE II: A severity of disease classification system. Crit Care Med 1985; 13: 18-829.

24. Beger HG, Bittner R, Silvia Block, Bqchler M. Bacterial contamination of pancreatic necrosis: a prospective clinical study. Gastroenterology 1986; 91:433-8.

25. Lece T, Shaw D. Comparison of three Glasgow multifactor prognostic scoring systems in acute pancreatitis. Br J Surg 1988; 75:460-463.

26. Fan ST, Lai EC, Mok FP. Early treatment of acute biliary pancreatitis by endoscopic papillotomy. N EnglJMed 1993; 328: 228.

27. Brisando G, Maria G, Ferrante A, Civello IM. Evaluation of prognostic factors in patients with acute pancreatitis. HepaGastroenterol 1999; 46: 1990-1997

28. Norman J. The Role of cytokines in the pathogenesis of acute pancreatitis. Am $\mathbf{J}$ surg 1998; 175: 76. 